\title{
Análise da morbidade materna grave em uma maternidade pública de Manaus no período de janeiro de 2018 a junho de 2019
}

\author{
Maternal morbidity of near miss in a public maternity hospital in Manaus from January \\ 2018 to June 2019
}

\author{
Mobilidade materna o cercana a la muerte en un hospital público de maternidad en \\ Manaus desde enero de 2018 hasta junio de 2019
}

\begin{abstract}
Kamila da Silva Atayde ${ }^{1 *}$, Raíssa Costa Said ${ }^{1}$, Marcello Facundo do Valle Filho², José Fernandes de Souza Viana'.
\end{abstract}

\section{RESUMO}

Objetivo: Discorrer sobre morbidade materna ou near miss em uma maternidade pública de Manaus. Métodos: Estudo retrospectivo desenvolvido em uma Maternidade pública de Manaus, obtidos por meio de prontuários médicos arquivados no serviço de arquivos médicos da maternidade. Resultados: Os resultados encontrados foram levantados por meio dos critérios e diagnósticos de near miss das pacientes admitidas na unidade de terapia intensiva da Maternidade que estavam no ciclo gravidicio-puerperal (grávidas, em trabalho de parto, que deram à luz ou abortaram dentro de um período de 42 dias) através de variáveis levantadas, cujas pacientes eram mulheres que apresentaram pelo menos um dos critérios de near miss segundo OMS. Conclusão: Evidenciou, entre as pacientes, uma frequência elevada de mulheres jovens, a grande maioria primigesta e que não tiveram assistência pré-natal adequada como preconizada pelo Ministério da Saúde, notou-se também os distúrbios hipertensivos relacionados a gestação e suas complicações como os principais critérios de near miss diagnosticados acometendo essas pacientes, ressaltando assim a importância de obter melhorias em relação a atenção primária em saúde.

Palavras-chave: Morbidade, Morte materna, Near miss.

\begin{abstract}
Objective: To discuss maternal morbidity or near miss in a public maternity hospital in Manaus. Methods: Retrospective study carried out at a public maternity hospital in Manaus, obtained through medical records filed at the maternity medical files service. Results: The results found were obtained through the criteria and diagnostics of near miss of the patients admitted to the intensive care unit of the Maternity who were in the pregnancy-puerperal cycle (pregnant women, in labor, who gave birth or miscarried within a period of 42 days) through variables raised, whose patients were women who had at least one of the near miss criteria according to WHO. Conclusion: Among the patients, there was a high frequency of young women, the vast majority of whom were primigravid and who did not have adequate prenatal care as recommended by the Ministry of Health, hypertensive disorders related to pregnancy and its complications, such as main criteria of near miss diagnosed affecting these patients, thus emphasizing the importance of obtaining improvements in relation to primary health care.
\end{abstract}

Key words: Morbidity, Maternal death, Near miss.

\section{RESUMEN}

Objetivo: Discutir la morbilidad materna o cuasi accidentes en una maternidad pública en Manaus. Métodos: estudio retrospectivo desarrollado en una maternidad pública de Manaus, obtenido a través de historias clínicas archivadas en el servicio de archivos médicos de maternidad. Resultados: Los resultados encontrados se obtuvieron a través de los criterios y diagnósticos de cuasi accidente de las pacientes ingresadas en la unidad de cuidados intensivos de la Maternidad que se encontraban en el ciclo embarazo-puerperal (gestantes, en trabajo de parto, que dieron a luz o abortaron en un lapso de tiempo). de 42 días) a través de variables planteadas, cuyos pacientes eran mujeres que tenían al menos uno de los criterios de cuasi accidente según la OMS. Conclusión: Entre las pacientes, hubo una alta frecuencia de mujeres jóvenes, la gran mayoría de las cuales eran primigrávidas

${ }^{1}$ Universidade do Estado do Amazonas (UEA), Manaus - AM. *E-mail: mila_atayde@hotmail.com

2 Instituto Metropolitano de Ensino (IME/FAMETRO), Manaus - AM 
y que no tenían la atención prenatal adecuada recomendada por el Ministerio de Salud, trastornos hipertensivos relacionados con el embarazo y sus complicaciones, como principales criterios de near miss diagnosticados que afectan a estos pacientes, destacando así la importancia de obtener mejoras en relación a la atención primaria de salud.

Palabras clave: Morbilidad, Muerte materna, Casi omisión.

\section{INTRODUÇÃO}

A mulher é um contínuo, oportuno e pertinente tema de interesse e de debate. A gravidez, apesar de ser um fenômeno fisiológico, acarreta em riscos de morbidade e mortalidade, mesmo sendo eventos raros. As evidências sobre intervenções benéficas no parto, adequado atendimento profissional durante a gestação, condições sociais adequadas e entre outros são fundamentais na determinação de bons resultados de saúde (CECATTI JG, 2005).

Compreende-se que a morte materna seja o fim de uma sequência de eventos que poderiam ter intervenções implementadas para evitar os casos de óbito. Muitas dessas intervenções são simples, de baixo custo e de fácil aplicação, levantando a reflexão do quanto os profissionais de saúde são fundamentais para ao desempenho e a qualidade de parte significativa dessas ações (ARAUJO LM, et al., 2021)

A quase morte (near miss) é um novo indicador de saúde, contribuindo para identificação dos fatores remediáveis do sistema de saúde, avaliação da qualidade do atendimento e orientação na tomada de decisões para gestores e profissionais da saúde. É importante citar a escassez de pesquisas envolvendo o near miss em gravidez gemelares, considerando que as condições socioeconômicas e demográficas maternas são possuem relação com maior índice desse tipo de gravidez (LOPES FNB, 2020).

A morte materna é estabelecida como qualquer morte ocorrida durante o período da gestação, parto ou até 42 dias de puerpério, por condições determinadas ou agravadas pela gravidez. É classificada como o pior resultado obstétrico, referente ao último evento de uma sequência que pode vir acontecer na gestação. $A$ maioria das gestações progredirá de uma forma saudável e fisiológica, finalizando-se com um parto sem intercorrências, gerando um conceito de peso e desenvolvimento adequado, com a plena recuperação da mulher ao fim do puerpério (SANTANA DS, et al., 2018).

No contexto mundial, a morte materna é utilizada como parâmetro para se avaliar a qualidade do serviço de saúde ofertado, identificando situações de desigualdade e contribuindo para a avaliação dos níveis de saúde e de desenvolvimento socioeconômico de uma população (LAURENTI R, et al., 2008). Existe uma parcela pequena de gestantes que são portadoras de alguma doença, sofrem algum agravo, problemas ou apresentam maiores probabilidades de evolução desfavorável para o feto e para a mãe, ainda considerando que a gestação é uma ocorrência fisiológica e sua evolução se dá sem intercorrências na maioria dos casos (BRASIL, 2010).

É relativamente recente o conceito de morbidade materna aguda grave ou near miss, portanto, não existe consenso sobre o critério operacional mais adequado. É dificil determinar o ponto a partir do qual se caracteriza a morbidade materna grave/near miss, porém, esta controvérsia pode ser atribuída ao amplo espectro de gravidade clínica: de um lado a gravidez saudável e de outro o óbito materno (MORSE ML, et al., 2011). É um episódio em que acontece a quase morte, ocasionado por complicações graves ocorridas com a mulher durante a gravidez, parto ou puerpério (SAY L, et al., 2009).

O near miss materno (NMM) é o ponto mais extremo da gravidade que antecede a morte, ou seja, a "quase" morte, e esta terminologia near miss foi adaptada pelas ciências médicas de um conceito usado na indústria aeronáutica que descreve um evento de quase colisão entre aeronaves por aproximação indevida, e que apenas não ocorreu por sorte ou por ação adequada (OLIVEIRA-NETO A, et al., 2009).

Portanto, sendo o ponto mais extremo do pior resultado obstétrico, é importante avaliar os principais critérios de morbidade materna grave, passando por conceitos e dados relevantes. O de near miss materno pode ser caracterizado pelas mulheres que sobrevivem a complicações graves relacionadas a gravidez, parto 
e pós-parto que possuem aspectos em comum com aquelas que morrem destas mesmas complicações (LONGHI SAT, 2019; PETERLINI OLG, 2019).

O perfil criado por Nogueira TF, et al. (2020) indica maior morbidade em adolescentes e predomínio de mulheres negras por reflexo de uma diferença sociodemográfica das gestantes, maior prevalência de gravidez nos extremos de idade (adolescência e acima de 35 anos), ausência de parceiro é um fator independente e a importância do pré-natal para evitar o pior resultado obstétrico (a morte). Boa parte das gestantes não completam todas as consultas necessárias, sendo uma das razões o fato delas iniciarem o pré-natal tardiamente, revelando dificuldades no acesso ao diagnóstico da gravidez e a necessidade de maior acessibilidade a assistência em saúde.

Além disso, Oliveira LC e Costa AAR (2015) destaca a elevada porcentagem de mulheres provenientes de municípios do interior do estado analisado, refletindo a precariedade da assistência prestada nestas localidades e a necessidade de melhorias. Além disso, destaca a baia qualidade nos serviços e a préeclampsia grave como principal diagnóstico associado ao near miss, coincidindo com outros estudos brasileiros. A principal causa de morte materna são as síndromes hipertensivas.

Mais ainda, é elevada a ocorrência de óbitos fetais e neonatais entre as pacientes com near miss materno. Claramente há uma sobreposição de fatores que contribuem para esse desfecho fatal, como pré-eclampsia grave, DPP, endometrite e parto prematuro ou critérios laboratoriais. Assim, reafirma-se a necessidade de melhorias na assistência pré-natal visando a identificação precoce dos fatores citados e adoção de medidas que evitem a progressão para complicações e minimizem as repercussões sobre a mãe e feto, ou seja, discutir o near miss e a mortalidade materna (OLIVEIRA LC e COSTA AAR, 2013).

Portanto, de acordo com o que fora relatado e a sua relevância para a saúde pública, este trabalho visa discutir a morbidade materna ou near miss analisando a população admitida na unidade de terapia intensiva em uma maternidade pública de Manaus e as respectivas variáveis que os quadros apresentam, como complicações, análise de pré-natal e número de gestações.

\section{MÉTODOS}

Trata-se de um estudo retrospectivo inserido na área de obstetricia, com a finalidade de avaliar os principais critérios de near miss ou morbidade materna grave em uma maternidade de grande porte do estado do Amazonas. A população foi constituída por pacientes admitidas na unidade de terapia intensiva da Maternidade, cujas pacientes eram mulheres que apresentaram pelo menos um dos critérios de near miss segundo a Organização Mundial da Saúde (OMS) e mulheres no ciclo gravídico-puerperal (Mulheres grávidas, em trabalho de parto, que deram à luz ou que abortaram dentro de um período de até 42 dias).

Não houve abordagem direta à população de interesse. As informações obtidas foram organizadas em uma ficha de coleta de dados proposta pelo desenho do estudo e posteriormente realizada a organização do percentual obtido pelos achados do trabalho. A ficha de dados continha informações a serem preenchidas baseadas no prontuário do paciente, bem como marcação das complicações, intervenções, disfunções ameaçadoras à vida, detalhes da morte materna, informações perinatais, status vital da criança, condições na chegada ao serviço e processo de encaminhamento, indicadores de processo, patologias associadas e dados do pré-natal.

O estudo foi submetido ao Comitê de Ética em pesquisa com seres humanos da Universidade Estadual do Amazonas sob número do CAAE: 13087519.7.0000.5016 e após aprovação foi dado ínicio a coleta de dados.

\section{RESULTADOS E DISCUSSÃO}

De acordo com os dados obtidos durante os meses de janeiro de 2018 á junho de 2019 em relação aos casos das gestantes que deram entrada na maternidade em Manaus, nota-se a distribuição segundo a faixa etária idade das mulheres que teve variação de 14 à 45 anos e que o percentual de maior incidência foi entre 14-20 anos equivalendo a 27,4\% da população estudada (Gráfico 1). 
Gráfico 1 - Distribuição por idade das mulheres atendidas em uma maternidade pública no período de junho/2018 a junho/2019.

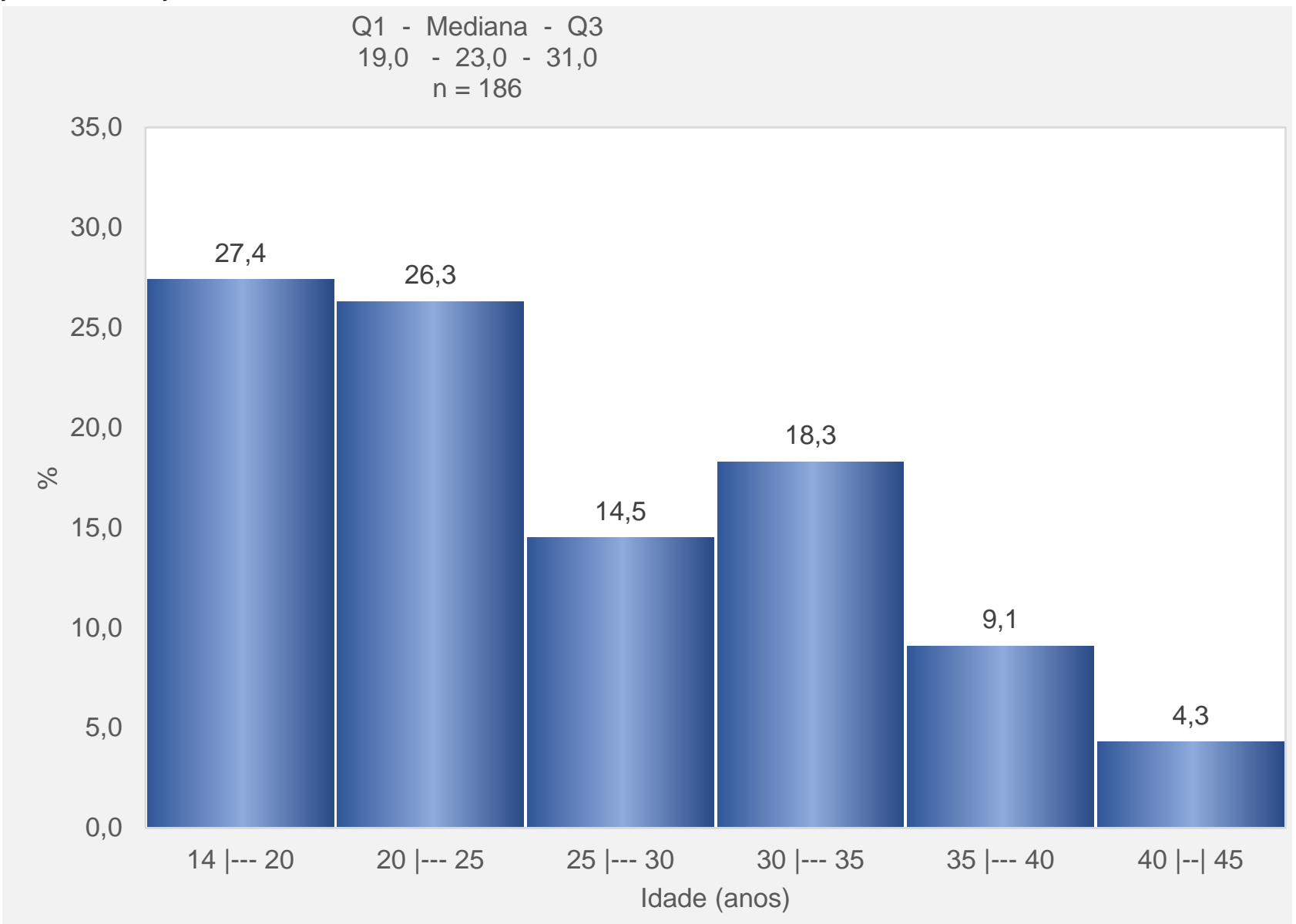

Fonte: Atayde KS, et al., 2020; dados extraídos de Serviço de Arquivo Médico e Estatística (SAME).

Em relação a frequência e número de consultas relacionadas ao pré natal, demostrou-se que das 186 pacientes, $57,5 \%$ fizeram o acompanhamento,enquanto $14,5 \%$ não o fizeram e $28 \%$ das pacientes não tiveram essa informação. Já em número de consultas das 107 pacientes o maior percentual foi de 44,9\% com uma quantidade de 4 a 6 consultas. A assistência pré-natal é vital, pois é nesse espaço de tempo que são estabelecidas e desenvolvidos trabalhos pautados na promoção da saúde (Tabela 1) (TOMASI E, et al., 2017).

Quando abordado o número de gestação, $48,4 \%$ das mulheres disseram ser a primeira gestação, em seguida de $17,7 \%$, já estavam na terceira gestação, e apenas $15,1 \%$ apresentando segunda gestação. Para pacientes com 4 gestações foram constatadas o equivalente a $4,8 \%$, para 5 gestações foram identificadas $7,0 \%$, e acima de 5 gestações o percentual foi de $7,0 \%$.

De acordo com o Ministério da Saúde (2017) a melhor assistência relevantes ao pré-natal devem ser realizadas no mínimo por seis consultas, sendo que destas seis pelo menos duas sejam realizadas por médico já no início no primeiro trimestre gestacional.

É necessário que se estabeleçam a educação em saúde, com o objetivo de levar a população a refletir sobre a necessidade do cuidado na gestação por meio do tratamento pré-natal. Nesse processo, deve haver a interação entre a gestante e o profissional de saúde, além de estender esse laço para com o pai e demais familiares a fim de que a confiança seja fortalecida durante a gestação e no pós-parto e dessa maneira seja promovido à adesão ao tratamento para que a saúde da mãe e da criança seja preservada (BRITO A, et al., 2008). Já quando verificado a idade gestacional em relação a semanas $50,5 \%$ apresentaram estar com valor menor ou igual a $\leq 35$. A média e o desvio padrão que obteve foi de $32,9 \pm 7,4$, e a amplitude foi de $0-42$. 
Tabela 1 - Frequência e número de consultas de pré-natal das mulheres atendidas em uma maternidade pública no período de janeiro/2018 a junho/2019.

\begin{tabular}{lcc}
\hline Diagnóstico $(\mathbf{n}=\mathbf{1 8 6})$ & $\mathbf{f i}$ & $\%$ \\
\hline Pré-natal & & \\
\hline Sim & 107 & 57,5 \\
Não & 27 & 14,5 \\
Sem informação & 51 & 28,0 \\
\hline
\end{tabular}

\begin{tabular}{lll}
\hline Número de consultas $(\mathbf{n}=\mathbf{1 0 7})$ & & \\
\hline 1 a 3 & 45 & 42,0 \\
4 a 6 & 48 & 44,9 \\
$>6$ & 14 & 13,1 \\
\hline
\end{tabular}

\begin{tabular}{|c|c|c|}
\hline Número de gestações & & \\
\hline 1 & 90 & 48,4 \\
\hline 2 & 28 & 15,1 \\
\hline 3 & 33 & 17,7 \\
\hline 4 & 9 & 4,8 \\
\hline 5 & 13 & 7,0 \\
\hline$>5$ & 13 & 7,0 \\
\hline Q1 - Mediana - Q3 & \multicolumn{2}{|c|}{$1-2-3$} \\
\hline Amplitude & \multicolumn{2}{|c|}{$1-12$} \\
\hline \multicolumn{3}{|c|}{ Idade gestacional (semanas) } \\
\hline$\leq 35$ & 94 & 50,5 \\
\hline$>35$ & 92 & 49,5 \\
\hline Média \pm Dp & \multicolumn{2}{|c|}{$32,9 \pm 7,4$} \\
\hline Amplitude & \multicolumn{2}{|c|}{$0-42$} \\
\hline
\end{tabular}

Fonte: Atayde KS, et al., 2020; dados extraídos de Serviço de Arquivo Médico e Estatística (SAME).

Sobre o diagóstico primário, pode-se observar que de acordo com os diagnósticos primários foi elevada a prevalência de eclampsia acometendo um percentual de $24,7 \%$, sendo seguido por pré-eclâmpsia grave atingindo cerca de $19,9 \%$. A eclâmpsia é o aparecimento de convulsão em uma paciente e pode ocorrer na gravidez, parto ou até 42 dias de puerpério. A iminência de eclâmpsia é caracterizada, clinicamente, por sinais de encefalopatia hipertensiva,náuseas, vômitos, dor em hipocôndrio direito e epigastralgia. Apesar da sua importância em saúde pública, a etiologia da hipertensão, que se manifesta na gestação (pré-eclâmpsia e hipertensão gestacional), permanece desconhecida (Tabela 2) (ZIEGEL EE, et al., 2004).

Na rede de saúde, a atenção ao diagnóstico secundário é formada pelos serviços especializados em nível ambulatorial e hospitalar, com densidade tecnológica intermediária entre a atenção primária e a terciária (BRASIL, 2010), historicamente interpretada como procedimentos de média complexidade. Esse nível compreende serviços médicos especializados, de apoio diagnóstico e terapêutico e atendimento de urgência e emergência. Os diagnósticos secundários achado no estudo foram de 16,1\%, apresentando diagnóstico secundário de sepse. Já para morte materna foram diagnosticado 2,7\%, para hemorragia pós-parto grave $2,2 \%$, para edema agudo de pulmão 1,1\%, e outras foram 3,8\%. enquanto para 138 pacientes $(74,8 \%)$, não apresentaram nenhum diagnóstico para diagnóstico secundário (Tabela 2). 
Tabela 2 - Frequência do diagóstico primário e secundário e médias do tempo de internação, número de gestações e idade gestacional das mulheres atendidas em uma maternidade pública no período de janeiro/2018 a junho/2019.

\begin{tabular}{lcc}
\hline Variáveis (n = 186) & $\mathbf{f}_{\mathbf{i}}$ & $\%$ \\
\hline Diagnóstico primário & & 24,7 \\
\hline Eclâmpsia & 36 & 19,9 \\
Pré-eclâmpsia grave & 27 & 14,5 \\
Infecção puerperal & 22 & 11,8 \\
Hemorragia pós-parto grave & 10 & 5,4 \\
Síndrome Hellp & 5 & 2,7 \\
Arritmia cardiac & 4 & 2,2 \\
H1N1 & 2 & 1,1 \\
Anemia grave & 2 & 1,1 \\
Diabetes gestacional & 2 & 1,1 \\
Erisipela & 2 & 1,1 \\
Hiperêmese gravídica & 2 & 1,1 \\
Sepse ou Infecção sistêmica & 2 & 1,1 \\
Trombose venosa profunda & 23 & 12,3 \\
Outros & & \\
\hline Diagnóstico secundário & 30 & 16,1 \\
\hline Sepse & 5 & 2,7 \\
Morte materna & 4 & 2,2 \\
Hemorragia pós-parto grave & 2 & 1,1 \\
Edema agudo de Pulmão & 7 & 3,8 \\
Outros & 138 & 74,2 \\
Nenhum & & \\
\hline Tempo de intrnaçao hospitalar (dias) & \\
\hline
\end{tabular}

Tempo de internação hospitalar (dias)

$\begin{array}{lc}\mathrm{Q}_{1}-\text { Mediana }-\mathrm{Q}_{3} & 5-8-13 \\ \text { Amplitude } & 0-66\end{array}$

Tempo de internação UTI (dias)

$\begin{array}{lc}\mathrm{Q}_{1}-\text { Mediana }-\mathrm{Q}_{3} & 2-3-6 \\ \text { Amplitude } & 0-42\end{array}$

Fonte: Atayde KS, et al., 2020; Dados extraídos de Serviço de Arquivo Médico e Estatística (SAME).

No estudo, pode-se avaliar que as variáveis em relação às complicações graves (condições potencialmente ameaçadora à vida) teve um índice maior relacionado à hemorragia pós-parto grave (HPP), evidenciando que das $82,8 \%$ não apresentaram evento no período 0 onde (não apresentaram complicações graves), enquanto $15 \%$ apresentaram evento no período 1 (que foi destinado a presença na chegada ou até 12 horas de internação) e 2,2\% no período 2 (após 12 hs) (Tabela 3).

A HPP é definida como a perda de sangue $\geq 500 \mathrm{ml}$ após o parto. Segundo a OMS, as referências para considerar a via de parto ao definir a HPP: $\geq 500 \mathrm{ml}$ após parto vaginal e $\geq 1000 \mathrm{ml}$ após cesariana (DAHLKE JD, et al., 2015). A HPP pode ser classifica como primária, quando acometida dentro das primeiras 24 horas do puerpério, secundária já conhecida como tardia, quando o sangramento tem início entre 24 horas e 12 semanas pós-parto (SILVA LGP e PAULA GM, 2014). 
Para o diagnóstico de Pré-eclâmpsia grave o achado no estudo para o período 0 (não estava presente complicações) foi de 79,6\%. Logo em seguida no período 1 (presente na chegada ou até 12 horas) 19,9\%, e um baixo percentual no período 2 (evento após as 12 hs) de 0,5\%. A pré-eclâmpsia grave foi o principal diagnóstico associado ao near miss, coincidindo com os achados de outros estudos brasileiros. Em contrapartida, Zanette E, et al. (2014), Em contrapartida, é uma pesquisa que se difere de pesquisas realizadas em países desenvolvidos, nos quais a hemorragia aparece em primeiro lugar (ZHANG WH, et al, 2005).

Das complicações de eclampsia $71 \%$ não apresentaram condições no tempo 0 (não estava presente complicações), enquanto no período 1:27,9\% demonstraram ter complicações presente na chegada ou até 12 horas, e somente 1,1\%, apresentaram evento após 12 hs. A Eclâmpsia é a manifestação convulsiva (tônico-clônicas generalizadas) ou comatosa da pré-eclâmpsia, quer de forma isolada ou associada à hipertensão arterial materna preexistente (SILVA V, et al., 2013).

Para as condições de Sepse ou infecção sistêmica grave, o presente estudo teve como valores de $78 \%$ no período 0 não estava presente complicações, $20,4 \%$ no período 1 e $1,6 \%$ no período 2 . A sepse ocorre em $16,9 \%$ das pacientes, porém em outro estudo brasileiro, seu resultado foi de $23,7 \%$. Apesar dessa enfermidade não aparecer entre as complicações mais frequentes, muitos estudos consideram-na como detentora de um maior índice de mortalidade $(7,4 \%)$, superando os distúrbios hemorrágicos $(2,8 \%)$ e hipertensivos $(0,4 \%)$ (ROOST M, et al., 2009). Por fim a rotura uterina do estudo apresentou dados equivalentes a $100 \%$ onde nenhuma apresentou evento de complicação.

As variáveis de intervenções críticas ou internação em UTI avaliadas foram: utilização de hemoderivados $67,7 \%$, onde não apresentaram evento no período 0 , um percentual de $28 \%$ com possíveis intervenções no período 1 (presente na chegada ou até 12 horas), e no período 2 (evento após as 12 hs) 4,3\% (Tabela 3).

De acordo com a International Commission on Radiation Protection (2000), a radiologia intervencionista é definida como aqueles "procedimentos que abrangem interferências diagnósticas e terapêuticas conduzidas por acesso percutâneo ou outros, comumente desempenhadas sob anestesia local e/ou sedação, utilizando imagem de fluoroscópica para utilizar a localização da lesão e o local de tratamento, e com isso monitorando o processo, controlando e documentando a terapia" $100 \%$ das pacientes do presente estudo não apresentaram este evento, relacionados a radiologia intervencionista.

Mais ainda, no mesmo estudo, em relação a disfunção orgânica (condições ameaçadoras à vida), os dados obtidos para as disfunção estudadas variavam entre $67,7 \%$ a $90,9 \%$ das pacientes, não apresentando evento no período 0 (não estava presente complicações) Já em relação a acontecimentos até 12 horas o valor achado foi de $5,9 \%$ a $31,2 \%$, referente a disfunção no período 1 (presente na chegada ou até 12 horas), e para o período 2 o percentual encontrado foi de $0,5 \%$ a 3,2\% sendo os eventos que ocorreram após 12 hs.

A análise feita referente a mortes maternas demonstraram um percentual de $96,8 \%$ no período 1 que a freqüência para morte durante a gravidez ou dentro de 42 horas após o término da gravidez foi de $96,8 \%$ no período 1 (pode sair) e apenas 1,6\% para ocorrência de evento que aconteceu em até 12 horas e após 12 horas. E para morte após 42 dias do término da gravidez não houve nenhuma ocorrência para os eventos $100 \%$ relatados.

As informações maternas e perinatais apresentam que, do total das 186 pacientes, o maior índice evidenciado foi para parto cesariana $75,8 \%$. Esse dado é semelhante à porcentagem de cesáreas nos estudos brasileiros, conforme A Rovig R, et al. (2015). Já o parto vaginal foi efetivo em 11,8\%, e 7,5\% foram mulheres liberadas com alta ou que morreram durante a gravidez (Tabela 4).

Muitos autores usaram como critérios diagnósticos de near miss materno a presença de eventos ou diagnósticos, como a pré-eclâmpsia, eclâmpsia, edema agudo de pulmão e tromboembolismo, conforme BRASIL (2006). Outros empregaram metodologias baseadas na ocorrência de eventos e/ou diagnósticos essencialmente obstétricos no ciclo grávido-puerperal, como pré-eclâmpsia, eclâmpsia, síndrome HELLP, hemorragia severa com perdas maiores que $1500 \mathrm{ml}$, transfusão ou mais, hemácias, rotura uterina e sepses grave (VANDECRUYS HI, et al., 2002). 
Tabela 3 - Frequência da complicações, intervenções, disfunção orgânica e mortes de mulheres atendidas em uma maternidade pública no período de janeiro/2018 a junho/2019.

\begin{tabular}{|c|c|c|c|c|}
\hline Variáveis $(n=186)$ & 0 & 1 & 2 & 3 \\
\hline \multicolumn{5}{|c|}{ Complicações graves (condições potencialmente ameaçadoras à vida) } \\
\hline Hemorragia pós-parto grave & 82,8 & 15,0 & 2,2 & - \\
\hline Pré-eclâmpsia grave & 79,6 & 19,9 & 0,5 & - \\
\hline Eclâmpsia & 71,0 & 27,9 & 1,1 & - \\
\hline Sepse ou infecção sistêmica grave & 78,0 & 20,4 & 1,6 & - \\
\hline Rotura uterine & 100,0 & - & - & - \\
\hline \multicolumn{5}{|l|}{ Intervenções críticas ou internação em UTI } \\
\hline Utilização de hemoderivados & 67,7 & 28,0 & 4,3 & - \\
\hline Radiologia intervencionista & 100,0 & - & - & - \\
\hline Laparotomia & 95,2 & 0,5 & 4,3 & - \\
\hline Internação em UTI & 0,5 & 93,0 & 6,5 & - \\
\hline \multicolumn{5}{|l|}{ Disfunção orgânica (Condições ameaçadoras à vida) } \\
\hline Disfunção cardiovascular & 82,2 & 16,7 & 1,1 & - \\
\hline Disfunção respiratória & 90,9 & 8,0 & 1,1 & - \\
\hline Disfunção renal & 79,6 & 19,9 & 0,5 & - \\
\hline Disfunção coagulação/hematológica & 67,7 & 31,2 & 1,1 & - \\
\hline Disfunção hepatica & 84,4 & 15,1 & 0,5 & - \\
\hline Disfunção neurológica & 69,9 & 29,0 & 1,1 & - \\
\hline Disfunção uterina/histerectomia & 90,9 & 5,9 & 3,2 & - \\
\hline \multicolumn{5}{|l|}{ Mortes maternas } \\
\hline $\begin{array}{l}\text { Morte durante a gravidez ou dentro de } 42 \text { horas após o } \\
\text { término da gravidez }\end{array}$ & 96,8 & 1,6 & 1,6 & - \\
\hline Morte após 42 dias do término da gravidez & 100,0 & - & - & - \\
\hline
\end{tabular}

Legenda: 0 = A condição não estava presente durante a hospitalização; 1 = Estava presente na chegada ou dentro de 12 horas da chegada ao hospital; 2 = Desenvolveu-se após 12 horas da chegada ao hospital; 3 = Informações não disponíveis/desconhecidas ou não aplicáveis.

Fonte: Atayde KS, et al., 2020; Dados extraídos de Serviço de Arquivo Médico e Estatística (SAME).

No Brasil, apesar da redução de 52\% nas taxas de mortalidade materna, de 120 óbitos maternos por 100 mil Nascidos Vivos (NV) em 1990 para 58/100 mil NV em 2008, a meta estipulada pelos Objetivos de Desenvolvimento do Milênio - 35 óbitos por 100 mil NV para o ano de 2015 - todavia não foi atingida (WHO, 2010).

Rosendo TMSS e Roncalli AG (2015), fizeram um levantamento referente ao ano de 2014, como estudo de base populacional realizado em Natal-RN e encontraram uma taxa de near miss de 41,1/1.000 NV. Já relatos de Oliveira LC e Costa AAR (2015) apurando estudo de outra pesquisa, realizada em Recife-PE, sobre dados de 225 prontuários de internações em unidade de terapia intensiva (UTI) durante os anos de 2007 a 2010, apresentou taxa de 12,8/1.000 nascidos vivos (NV). 
Tabela 4 - Frequência de informações maternas, estimativa da idade gestacional, status vital da criança e condições de chega ao serviço por parte das mulheres atendidas em uma maternidade pública no período de janeiro/2018 a junho/2019.

\begin{tabular}{lcc}
\hline Variáveis (n = 186) & $\mathbf{f}_{\mathbf{i}}$ & $\%$ \\
\hline Informações maternas e perinatais & 22 & 11,8 \\
\hline Parto vaginal & 141 & 75,8 \\
Cesariana & 1 & 0,5 \\
Abortamento complete & 7 & 3,8 \\
Curetagem/aspiração a vácuo & 14 & 7,5 \\
Mulheres liberadas com alta ou que morreram durante a & 1 & 0,5 \\
gravidez & 1 & \\
\hline
\end{tabular}

\section{Melhor estimativa da idade gestacional em semanas completas}

\begin{tabular}{|c|c|c|c|c|}
\hline $\begin{array}{l}\text { Parto ou abortamento }(\mathrm{n}=171) \\
\text { Média } \pm \mathrm{Dp} \\
\text { Amplitude } \\
\text { Morte materna ou alta hospitalar }(\mathrm{n}=171) \\
\text { Média } \pm \mathrm{Dp} \\
\text { Amplitude }\end{array}$ & & & & \\
\hline \multirow{2}{*}{ Em relação ao status vital da criança $(n=171)$} & \multicolumn{2}{|c|}{0} & \multicolumn{2}{|c|}{1} \\
\hline & fi & $\%$ & fi & $\%$ \\
\hline Ao nascer & 142 & 83 & 29 & 17 \\
\hline $\mathrm{Na}$ alta hospitalar ou no $7^{\circ}$ dia de vida & 142 & 83 & 29 & 17 \\
\hline Sem informação & 15 & - & - & - \\
\hline \multicolumn{5}{|c|}{$\begin{array}{l}\text { Em relação às condições na chegada ao serviço de saúde e ao processo de encaminhamento } \\
(n=186)\end{array}$} \\
\hline $\begin{array}{l}\text { O parto ou abortamento ocorreu antes da chegada a } \\
\text { qualquer serviço de saúde }\end{array}$ & 180 & 96,8 & 6 & 3,2 \\
\hline Parto dentro de 3 horas da chegada ao serviço de saúde & 126 & 67,7 & 60 & 32,3 \\
\hline $\begin{array}{l}\text { Laparotomia dentro de } 3 \text { horas da chegada ao hospital ou } \\
\text { em outro hospital }\end{array}$ & 165 & 88,7 & 21 & 11,3 \\
\hline Mulher encaminhada de outro serviço de saúde & 99 & 53,2 & 87 & 46,8 \\
\hline $\begin{array}{l}\text { Mulher encaminhada a qualquer hospital de maior } \\
\text { complexidade }\end{array}$ & 154 & 82,8 & 32 & 17,2 \\
\hline
\end{tabular}

Legenda: $f_{i}=$ frequência absoluta simples; $D p=$ desvio-padrão, [0] NÃO [1] SIM.

Fonte: Atayde KS, et al., 2020; Dados extraídos de Serviço de Arquivo Médico e Estatística (SAME).

Dados mostram que a frequência para prevenção de hemorragia pós-parto a utilização da ocitocina foi de $87 \%$, enquanto para a utilização de outro uterotônico foi de 1,0\%, no período 1 (estava presente na chegada, dentro de 12 horas ou após 12 horas da chegada ao hospital).

O tratamento de hemorragia pós-parto que foi abordado no estudo demonstrou que a maior frequência encontrada foi de 22,5\% para ocitocina, para ergometrina foi de 17,7\%, o mesmo encontrado para misoprostol, e os demais apresentaram percentual inferior a 8,6\% (Tabela 5).

Os anticonvulsivantes que mostraram que $38 \%$ das pacientes utilizaram sulfato de magnésio, enquanto outros anticonvulsivantes apresentaram utilização que se refere a um percentual de 18 \%.

Para a utilização de antibiótico foi utilizado o antibiótico profilático durante a cesariana foi de62,4\%, enquanto para antibióticos terapêuticos, parenterais foi de $40 \%$. Para achados relacionados a maturação pulmonar fetal abordada foi de Corticosteroides (betametasona ou dexametasona), a frequência foi de 17,7\%.

Para as causas subjacentes ao óbito/Near Miss, os achados de maior evidência foram apresentados em frequência para transtornos hipertensivos foi de 49,5\%, seguido da infecção relacionada à gestação (20\%), e outra doença ou complicação obstétrica apresentando percentual de 18,8\%, no período 1 (estava presente na chegada, dentro de 12 horas ou após 12 horas da chegada ao hospital). O percentual levantado para patologias associadas/contribuintes para anemia foi de 38\%, cesárea anterior 14\%, e parto prolongado/obstruído $1,5 \%$. 
Tabela 5 - Frequência da prevenção, tratamento, medicação, Near Miss e patologias das mulheres atendidas em uma maternidade pública no período de janeiro/2018 a junho/2019.

\begin{tabular}{|c|c|c|c|c|}
\hline \multirow{2}{*}{ Variáveis $(n=186)$} & fi & $\%$ & fi & $\%$ \\
\hline & \multicolumn{2}{|c|}{0} & \multicolumn{2}{|c|}{1} \\
\hline \multicolumn{5}{|l|}{ Prevenção de hemorragia pós-parto } \\
\hline Ocitocina & 24 & 13 & 162 & 87 \\
\hline Outro uterotônico & 184 & 99 & 2 & 1 \\
\hline \multicolumn{5}{|l|}{ Tratamento de hemorragia pós-parto } \\
\hline Ocitocina & 144 & 77,4 & 42 & 22,5 \\
\hline Ergometrina & 153 & 82,3 & 33 & 17,7 \\
\hline Misoprostol & 153 & 82,3 & 33 & 17,7 \\
\hline Outros úterotônicos & 177 & 95 & 9 & 5 \\
\hline Ácido tranexâmico & 170 & 91,4 & 16 & 8,6 \\
\hline Remoção de produtos retidos & 185 & 99,5 & 1 & 0,5 \\
\hline Ligadura arterial (uterina/hipogástrica) & 184 & 99 & 2 & 1 \\
\hline Histerectomia & 173 & 93 & 13 & 7 \\
\hline \multicolumn{5}{|l|}{ Anticonvulsivantes } \\
\hline Sulfato de magnésio & 115 & 62 & 71 & 38 \\
\hline Outros anticonvulsivantes & 152 & 82 & 34 & 18 \\
\hline \multicolumn{5}{|l|}{ Antibióticos } \\
\hline Antibiótico profilático durante a cesariana & 70 & 37,6 & 116 & 62,4 \\
\hline Antibióticos terapêuticos, parenterais & 112 & 60 & 74 & 40 \\
\hline \multicolumn{5}{|l|}{ Maturação pulmonar fetal } \\
\hline $\begin{array}{l}\text { Corticosteroides (betametasona ou } \\
\text { dexametasona) }\end{array}$ & 153 & 82,3 & 33 & 17,7 \\
\hline \multicolumn{5}{|l|}{ Causas subjacentes ao óbito/Near Miss } \\
\hline Gestação terminada em aborto & 183 & 98,5 & 3 & 1,5 \\
\hline Hemorragia obstétrica & 155 & 83,5 & 31 & 16,5 \\
\hline Transtornos hipertensivos & 94 & 50,5 & 92 & 49,5 \\
\hline Infecção relacionada à gestação & 149 & 80 & 37 & 20 \\
\hline Outra doença ou complicação obstétrica & 151 & 82 & 35 & 18 \\
\hline $\begin{array}{l}\text { Doença ou complicação } \\
\text { médica/cirúrgica/mental }\end{array}$ & 173 & 93 & 13 & 7 \\
\hline Patologias concomitants & 165 & 89 & 21 & 11 \\
\hline \multicolumn{5}{|l|}{ Patologias associadas/contribuintes } \\
\hline Anemia & 116 & 62 & 70 & 38 \\
\hline \multicolumn{5}{|l|}{ Infecção por HIV } \\
\hline Cesárea anterior & 160 & 86 & 26 & 14 \\
\hline Parto prolongado/obstruído & 183 & 98,5 & 3 & 1,5 \\
\hline
\end{tabular}

Legenda: 0 = A condição não estava presente durante a hospitalização; 1 = Estava presente na chegada, dentro de 12 horas ou após 12 horas da chegada ao hospital

Fonte: Atayde KS, et al., 2020; Dados extraídos de Serviço de Arquivo Médico e Estatística (SAME).

\section{CONCLUSÃO}

Entre as pacientes com near miss materno, uma frequência elevada de mulheres jovens, a grande maioria primigesta e que não tiveram assistência pré-natal adequada como preconizada pelo Ministério da Saúde, notou-se também os distúrbios hipertensivos relacionados a gestação e suas complicações como os principais critérios de near miss diagnosticados acometendo essas pacientes, ressaltando assim a importância de obter 
melhorias em relação a atenção primária em saúde dando enfoque ao pré-natal, afim de buscar um índice maior de acompanhamento em relação as gestantes de faixa etária mais novas, ou de primeira gestação, com ampla assistência ao binômio materno-fetal desde a suas primeiras semanas e assim conseguir obter diagnósticos mais eficazes ou até mesmo intervir em situações que possam vir desencadear ao longo da gestação, minimizando assim os possíveis desfechos desfavoráveis na gestação.

\section{REFERÊNCIAS}

1. ARAUJO LM, et al. Health promotion actions and maternal near miss: an integrative review. Rev Rene, 2021; 22:e60394.

2. BRASIL, Ministério da Saude. Secretaria de Atenção a Saúde. Departamento de Ações: manual técnico. 5. ed. Brasília: Editora do Ministério da Saúde, 2010:302.

3. DAHLKE JD, et al. Prevention and management of postpartum hemorrhage: a comparison of 4 national guidelines. Am J Obstet Gynecol. 2015;213(1):76e 1-10.

4. LAURENTI R, et al. Mortes maternas e mortes por causas maternas. Epidemiol Serv Saúde, 2008; 17(4): $283-92$.

5. LONGHI SAT, et al. Comissão interna de near miss materno em um hospital da rede mãe paranaense no sudoeste do paraná: implantação, desafios e perspectivas R. Saúde Públ. Paraná, 2019.;2(Suppl 1):21-30

6. LOPES FNB. Fatores associados ao Near Miss materno e neonatal em gestações gemelares. Faculdade de Medicina, Universidade Federal do Ceará, Fortaleza, 2020.

7. MORSE ML, et al. Morbidade Materna Grave e Near Misses em Hospital de Referência Regional. Ver Bras Epidemiol., 2011;14 (2).

8. NOGUEIRA TF, et al. Near miss materno: perfil dos casos em hospital de referência em saúde materno infantil. Instituo de Medicina Integral Prof. Fernando, Figueira, IMIP, 2020.

9. OLIVEIRA LC, COSTA AAR. Óbitos fetais e neonatais entre os casos de near miss materno. Revista da Associação Médica Brasileira, 2013; 59(5).

10. OLIVEIRA LC, COSTA AAR. Near miss materno em unidade de terapia intensiva: aspectos clínicos e epidemiológicos. Rev Bras Ter Intensiva, 2015;27(3):220-7.

11. OLIVEIRA-NETO A, et al. Evaluation of the Sequential Organ Failure Assessment (SOFA) score in quantifying organ failure and outcome in severe maternal morbidity in obstetric intensive care. Unpublished, 2009.

12. ROSENDO TMSS, RONCALLI AG. Prevalência e fatores associados ao Near Miss Materno: inquérito populacional em uma capital do nordeste Brasileiro. Cienc Saude Coletiva, 2015; 20(4):1295-30

13. SANTANA DS, et al. Near miss materno - entendendo e aplicando o conceito. Rev Med., 2018;97(2):187-94.

14. SAY L, et al. WHO working group on Maternal Mortality and Morbidity Classifications. Maternal near miss-towards a standard tool for monitor-ing quality of maternal health care. Best Practice \& Research Clinical Obstetrics \& Gynaecology, 2009,23:287-296.

15. SILVA LGP, PAULA GM. Hemorragia pós-parto. In: Rezende J, Montenegro CAB. Rezende: Obstetrícia fundamental. $13 a$ ed. Rio de Janeiro: Guanabara Koogan, 2014; 881-5.

16. VANDECRUYS HI, et al. Severe acute maternal morbidity and mortality in the Pretoria Academic Complex: changing patterns over 4 years. Eur J Obstet Gynecol Reprod Biol., 2002; 102(1):6-10.

17. WHO. World Healh Organization. Guia para a Documentação e Partilha das "Melhores Práticas" em Programas de Saúde. Rwanda: Escritório Regional Africano Brazzaville/ OMS, 2008.

18. ZANETTE E, et al. Brazilian Network for Surveillance of Severe Maternal Morbidity Group. Maternal near miss and death among women with severe hypertensive disorders: a Brazilian multicenter surveillance study. Reprod Health, 2014;11(1):4.

19. ZHANG WH, et al. MOMS-B Group. Incidence of severe pre-eclampsia, postpartum haemorrhage and sepsis as a surrogate marker for severe maternal morbidity in a European population-based study: the MOMS-B survey. BJOG, 2005;112(1):89-96.

20. ZIEGEL EE, et al. Enfermagem Obstétrica. 8ª ed. Guanabara Koogan: Rio de Janeiro, 2004. 